\title{
Hochbegabung und Savant-Syndrom - eine Superioritätspathologie?
}

Sabine Müller und Jan Steinmetzer

\section{Einleitung}

Welche Merkmale Hochbegabte auszeichnen, was die Ursachen ihrer besonderen Fähigkeiten sind und ob diese notwendigerweise mit spezifischen Defiziten verbunden sind, wird in der Wissenschaft kontrovers diskutiert. Das liegt zum einen daran, dass unter dem Begriff „Hochbegabte“ eine sehr heterogene Personengruppe zusammengefasst wird: erstens die Hochbegabten im Sinne der modernen Psychologie, die einen IQ von mindestens 130 sowie Leistungsorientierung und intellektuelles Interesse besitzen; ${ }^{1}$ zweitens die „Wunderkinder“, also Kinder, die ein besonderes Talent und einen deutlichen Entwicklungsvorsprung in einem oder mehreren intellektuellen, musischen, künstlerischen oder sportlichen Bereichen haben, drittens die sog. Savants (früher: idiots savants), die über Spezialhochbegabungen verfügen und zugleich retardiert oder geistig behindert sind. Gemeinsam sind fast allen Hochbegabten das extrem frühe Auftreten der besonderen Begabung, ein exzessiver Wissensdurst sowie ein besonders hohes Niveau in bestimmten Bereichen und Disziplinen. Höchstwahrscheinlich sind alle Arten von Hochbegabung hirnorganischen Ursprungs; durch Stimulation und Training können sie jedenfalls nicht erreicht werden. Zwar lassen sich durch intensive Frühförderung enorme Leistungen bei Kindern erreichen, die die ihrer Altersgenossen weit übertreffen, doch diese „gemachten“ Wunderkinder sind keine Hochbegabten im eigentlichen Sinn.

1 Hochbegabt in diesem Sinne sind ca. 2 \% der Bevölkerung. Vgl. Winner (1998), S. 31. 
In der Wissenschaft wird Hochbegabung bis heute als ambivalent betrachtet und häufig im Zusammenhang mit psychosozialen Abweichungen oder bestimmten Krankheitsdispositionen gesehen. Bei den sog. Savants besteht tatsächlich ein Zusammenhang mit bestimmten psychischen Behinderungen, insbesondere mit Autismus oder dem Asperger-Syndrom. Aber auch bei universell Hochbegabten scheinen Zusammenhänge mit psychischen Störungen und sozialen Schwierigkeiten zu bestehen.

In diesem Aufsatz gehen wir der Frage nach, warum und unter welchen Umständen Hoch- und Spezialbegabungen ins Blickfeld der Medizin gerieten und als pathologisch angesehen wurden. Wir untersuchen insbesondere den Zusammenhang von spezifischen Hochbegabungen mit typischen Defiziten und diskutieren die Hypothese, dass extreme Begabungen auf einer Superioritätspathologie basieren als Folge bestimmter Hirnläsionen (Kapitel 2). Anschließend wagen wir einen Blick in die Zukunft: auf zukünftige medizinische Möglichkeiten zur künstlichen Erschaffung von Hoch- und Spezialbegabungen (Kapitel 3).

\section{Hochbegabung aus der Perspektive der Medizin}

Dass Hochbegabte aufgrund ihrer besonderen Fähigkeiten besondere Beachtung und Wertschätzung erfuhren, ist nur die eine Seite der Medaille. Die andere ist, dass Hochbegabung als in eklatanter Weise von der Norm abweichend betrachtet wird und daher immer wieder in den Verdacht gerät, irgendwie pathologisch zu sein. Dieser Verdacht äußert sich in unterschiedlicher Weise: (1) als Befürchtung, hochbegabte Menschen, vor allem hochbegabte Kinder, seien besonders krankheitsanfällig und kurzlebig; (2) als Meinung, hochbegabte, vor allem kreative Menschen seien in sozialer Hinsicht problematisch, psychisch gestört oder sogar wahnsinnig; (3) als wissenschaftliche Hypothese über eine Verknüpfung von Hochbegabung mit bestimmten Formen geistiger Behinderung aufgrund bestimmter hirnorganischer Besonderheiten.

Für diese Vermutungen gibt es eine Reihe Belege - aber auch für deren Cegenteil.

\subsection{Besondere Vulnerabilität von Hochbegabten?}

Seit dem 18. Jahrhundert haben Ärzte vermehrt Empfehlungen zur adäquaten Behandlung von hochbegabten Kindern gegeben. Viele Mediziner in der Epoche der Aufklärung waren über die physische Verfassung hochbegabter Kinder besorgt. ${ }^{2}$

Es wurde zum einen angenommen, dass hochbegabte Kinder besonders kränklich und schwächlich seien, zum anderen, dass sie durch die frühzeitigen Leistungen überfordert und vorzeitig verbraucht seien: „Early ripe, early

2 Z. B. äußerte sich der Arzt Johann Storch (1681-1751) folgendermaßen: „Meist freuen sich die Eltern dieser Kinder, weil sie noch im kindlichen Alter zur Doktorwürde gelangen. Aber die meisten Hoffnungen trügen: Es läuft darauf hinaus, daß dem Leibe die nötigen Lebenskräfte entzogen werden und derselbe schwach und kränklich werden muß." - Storch (1751), S. 178. 
rot. " ${ }^{3}$ Hartnäckig hielt sich auch die These, dass Hochbegabte eine geringere Lebenserwartung hätten. ${ }^{4}$ Sie wurde erst in der jüngeren Vergangenheit als unzutreffend entlarvt. Empirische Untersuchungen scheinen eher das Gegenteil zu belegen: Eine hohe Intelligenz scheint eher mit guter psychischer und physischer Gesundheit zu korrelieren als mit besonderer Vulnerabilität. ${ }^{5} \mathrm{Ob}$ dies auch für Extremformen, insbesondere für einseitige Hochbegabungen gilt, ist durch diese Studien allerdings noch nicht beantwortet.

Der Evolutionspsychologe Geoffrey Miller (2001) hält hohe Intelligenz für ein Indiz für ein gutes Gehirn und damit für insgesamt gute Gene. Für diese These könnte auch der Befund sprechen, dass höhere Intelligenz mit einer höheren Geschwindigkeit der Informationsverarbeitung ${ }^{6}$ korreliert ist, insbesondere mit dem Alpha-Rhythmus des EEG. Personen mit gutem Gedächtnis und hoher Intelligenz weisen eine lokalisiertere Aktivierung (Alpha-Unterdrückung) auf als andere. Eine effiziente, d. h. lokalisiertere Aktivierung scheint ein wichtiges Merkmal für Intelligenzleistung zu sein. Dies zeigt sich auch in PET-Untersuchungen: Intelligentere Versuchspersonen weisen beim Lösen leichterer Aufgaben eine signifikant geringere metabolische Aktivierung und beim Lösen schwierigerer Aufgaben eine selektivere Aktivierungszunahme in der dafür relevanten Gehirnhemisphäre auf als weniger intelligente Personen. Die Gehirne intelligenterer Menschen müssen sich also beim Lösen leichter Aufgaben weniger anstrengen und können ihren Stoffwechsel für das Lösen schwierigerer Aufgaben besser aktivieren. ${ }^{7}$ Einige biologisch orientierte Intelligenzforscher betrachten die Informationsverarbeitungsgeschwindigkeit als basale, stark genetisch bestimmte Eigenschaft. Die Gehirne intelligenter Menschen antworten schneller und mit komplexeren EEG-Wellenformen auf äußere Reize. Demnach bestimmt die neuronale Effizienz des Zentralnervensystems, also die Schnelligkeit, Zuverlässigkeit und Konsistenz der Impulsübertragung, das Intelligenzniveau. ${ }^{8}$

\subsection{Genie und Wahnsinn? Psychosoziale Schwierigkeiten von Hochbegabten?}

Schon in der Antike wurde darüber spekuliert, inwieweit Genie und Wahnsinn miteinander in Verbindung stehen. Auguste Tissot (1728-1797) nahm eine Beziehung von kindlicher Hochbegabung und Wahnsinn im Erwachsenenalter an. Im 19. Jahrhundert begannen Psychiater wie Cesare Lombroso (1836-1909) Genialität mit „Irrsinn“ in Verbindung zu bringen: Der das Genie überfallen-

3 Winner (1998), S. 190.

4 Nissen (2005), S. 67 ff., bzw. Oehme (1988), S. $109 \mathrm{ff}$.

5 Vgl. Deary/Der (2005); Rudinger/Rietz (1997).

6 Unter der Informationsverarbeitungsgeschwindigkeit wird die Verarbeitungszeit für ganz einfache Reize verstanden.

7 Vgl. Klimesch/Schimke (1998), S. 150-156. - Personen mit guter Gedächtnisleistung zeigen eine um ca. $1 \mathrm{~Hz}$ höhere Alpha-Frequenz als Versuchspersonen mit schlechter Gedächtnisleistung. (Die Alpha-Frequenz liegt bei Menschen in einem Bereich von 7 bis $13 \mathrm{~Hz}$.) Der Alpha-Rhythmus hat in Ruhe eine höhere Amplitude als bei geistiger Anstrengung. Dieses als Alpha-Unterdrückung bezeichnete Phänomen weist eine topographisch spezifische Verteilung auf, die von der Art der bewältigten kognitiven Aufgabe abhängt.

8 Vgl. Guthke (1996), S. 94-103. - Unter der Informationsverarbeitungsgeschwindigkeit wird die Verarbeitungszeit für ganz einfache Reize verstanden. - Vgl. zu diesem Abschnitt Müller (2008). 
de Schaffensdrang habe Ähnlichkeit mit den manischen Phasen bei bipolarer Depression oder anderen psychischen Störungen. Lombrosos Theorie hatte einen nachhaltigen Einfluss: Noch in den $1920 e r$ Jahren beriefen sich mehrere Autoren wie Wilhelm Lange-Eichbaum ${ }^{9}$ (1875-1950) und Ernst Kretschmer ${ }^{10}$ (1888-1964) auf die These, dass Genialität mit psychopathologischen Phänomenen in Zusammenhang stehe. ${ }^{11}$ Erst seit kurzem hat die Psychiatrie diese Theorie fallen gelassen.

Das Klischee von Genie und Wahnsinn trifft am ehesten noch auf Künstler und Schriftsteller zu; bei ihnen sind Unzufriedenheit, Oppositionalität und affektive Probleme, vor allem eine leichte manische Depression, tatsächlich der Kreativität förderlich. ${ }^{12}$

Aber auch die moderne Hochbegabungsforschung konstatiert bei Hochbegabten bestimmte Eigenschaften, durch die sie vermehrt psychische Probleme und soziale Schwierigkeiten haben sollen. ${ }^{13}$ Da hochbegabte Kinder besonders sensibel sind, sind sie psychisch anfälliger. ${ }^{14}$ Wegen ihrer Frühreife und Andersartigkeit ziehen hochbegabte Kinder das Alleinsein oder die Gesellschaft Älterer vor. Sie interessieren sich für philosophische und moralische Fragen und entwickeln eigene, oft radikale Standpunkte. Deswegen und weil sie Geschlechtsstereotypen meist ablehnen, kritisch gegenüber Lehrern und häufig nonkonformistisch, risikobereit und oppositionell sind, haben sie häufiger soziale Schwierigkeiten und ein geringeres Selbstvertrauen in die eigenen sozialen Fähigkeiten als andere Kinder und sind daher häufiger einsam. ${ }^{.5}$ In zeitgenössischen Publikationen zu Hochbegabten werden die sozialen Schwierigkeiten hochbegabter Kinder sowie das absichtliche Versagen, um von Gleichaltrigen akzeptiert zu werden, besonders thematisiert. ${ }^{16}$

Andererseits relativieren neuere Untersuchungen die These, dass mit Hochbegabung soziale Schwierigkeiten verbunden sein müssen: Zwar haben Kinder mit einem IQ über 160 große soziale Schwierigkeiten, doch Kinder mit IQ-Werten zwischen 125 und 155 sind demnach i. a. emotional ausgeglichen und sozial

9 Lange-Eichbaum wies 1928 darauf hin, dass es einer „Verehrergemeinde“ bedürfe, die eine Hochleistung zur Leistung eines Genies erkläre. Da zwischen der Leistung selbst und ihrer Anerkennung indessen meist ein sehr langer Zeitraum liege, ergäben sich zwangsläufig für jedes Genie Probleme, die oft zu erheblichen sozialen und gesundheitlichen Belastungen führten. Verehrer fänden sich allerdings leichter, wenn es um keine normale Leistung gehe, sondern um etwas Ungewöhnliches, ja geistig Unnormales oder Krankhaftes. - Vgl. Lange-Eichbaum (2000), S. 134.

10 Kretschmer (1929).

11 Sie standen damit in einer langen geistesgeschichtlichen Tradition: Zum ersten Mal hatte Georg Christoph Lichtenberg im Bemühen, die Physiognomie Lavaters zu widerlegen, einen stringenten Zusammenhang zwischen Genialität, Kriminalität und Irrsinn apostrophiert; vgl. Hagner (2007), S. 19.

12 Vgl. Winner (1998), S. 265-274; Goleman (1998), S. 114. - Zur Kreativität vgl. Csikszentimihalyi (1996); Eysenck (1995); Gardner (1993); Howe (2001); Ross (2007).

$13 \mathrm{Vgl}$. zu hochbegabten Kindern und ihren Persönlichkeitsmerkmalen sowie besonderen psychischen oder sozialen Problemen: Feger (1988); Feger/Prado (1998); Fortenbacher (2006); Freund-Braier (2000); Pruisken (2005); Reichle/Lehmann/Jüling (2004); Rost (1993); Schulte zu Berge (2001); Schütz (2004); Sparfeldt (2006); Urban (2004); Vom Scheidt (2004); Winner (1998).

14 Vgl. Brackmann (2005); Winner (1998), S. 34 und 206.

15 Vgl. Winner (1998), S. 196-214.

16 Vgl. z. B. Schilling (2002); Vom Scheidt (2004); Winner (1998), S. 192-214. 
gut integriert. ${ }^{17}$ Hochbegabte Jungen sind im Durchschnitt beliebter als normal begabte, während es bei Mädchen umgekehrt ist; das liegt wahrscheinlich daran, dass die für Hochbegabte typischen Merkmale Leistungsbereitschaft und Unabhängigkeit gegen das weibliche Rollenklischee verstoßen. ${ }^{18}$

Aus Langzeitstudien, insbesondere der Terman-Studie, in denen hochbegabte Kinder und Jugendliche bis ins mittlere Erwachsenenalter begleitet und wiederholt befragt wurden, ergibt sich, dass der überwiegende Teil von ihnen in Studium und Beruf überdurchschnittlich erfolgreich ist. ${ }^{19}$ Die Studien zeigen aber auch, dass eine hohe intellektuelle Begabung allein hierfür nicht ausreicht, sondern dass viele weitere Faktoren darüber mitbestimmen: neben familiärer und schulischer Unterstützung vor allem die Leistungsmotivation sowie bestimmte Persönlichkeitsmerkmale. Erfolgsbegünstigende Merkmale sind Selbstvertrauen, Energie, Konzentrationsfähigkeit, souveränes Auftreten, Unabhängigkeit und Risikobereitschaft. ${ }^{20}$

\subsection{Superioritätspathologie als Grundlage von Hoch- und Spezialbegabungen ?}

Bei Savants besteht ein enger Zusammenhang zwischen ihren besonderen Fähigkeiten und ihren speziellen Einschränkungen, der hirnorganische Besonderheiten vermuten lässt. Savants verfügen über Hochbegabungen auf eng umgrenzten Gebieten, sind aber stark retardiert, meist unfähig, ein selbständiges Leben zu führen, und häufig autistisch.

Oliver Sacks berichtet von zwei Zwillingsbrüdern: autistische, frühgeborene Savants mit einem IQ von 6o, aber mit einem extremen Zahlengedächtnis und der Fähigkeit zur Kalenderberechnung (Berechnung des Wochentags zu beliebigen Daten) sowie zur Bestimmung zwölfstelliger Primzahlen - und das, obwohl sie die Grundrechenarten nur rudimentär beherrschen. Darüber hinaus verfügen sie über ein eidetisches Gedächtnis, in dem die winzigsten visuellen Details und alle biographischen Details festgehalten werden, die sie aber ohne irgendeine emotionale Beteiligung preisgeben. Sacks nimmt an, dass der Schlüssel ihrer Fähigkeiten eine Visualisierung von enormer Intensität, grenzenloser Ausdehnung und absoluter Exaktheit sei; dass sie gewissermaßen eine „riesige Erinnerungstapete“ besäßen, auf der sie Primzahlen und Kalender sehen, statt sie zu berechnen. ${ }^{22}$ Tatsächlich scheinen die Stärken der Savants zugleich ihre Schwächen zu sein: Sie sehen den Wald vor lauter Bäumen nicht. ${ }^{23}$

Vilayanur Ramachandran ${ }^{24}$ berichtet von kindlichen Savants, die lebensechte Zeichnungen komplexer Szenen produzieren, die den Vergleich mit de-

17 Vgl. Winner (1998), S. 208.

18 Vgl. Winner (1998), S. 212.

19 Vgl. Winner (1998), S. 257-261.

20 Vgl. Winner (1998), S. 265-274.

21 Vgl. zu diesem Kapitel Müller (2008).

22 Vgl. Sacks (1990), S. 246-278. - Vgl. zu Kalenderrechnern, insbes. den Zwillingen, auch Treffert (1989), S. 36-54.

$23 \mathrm{Vgl}$. Hein (2003).

24 Vgl. zu diesem Abschnitt Ramachandran/Blakeslee (2002), S. 283-320. 
nen von Leonardo da Vinci nicht zu scheuen brauchen, sowie von Savants, die jederzeit die Tageszeit oder die Breite von Gegenständen exakt bestimmen können. Er berichtet außerdem über den Inder Srinivasan Ramanujan, der aus einfachen Verhältnissen stammte, keinerlei Ausbildung in höherer Mathematik hatte, aber bedeutende Entdeckungen in der Zahlentheorie machte. Das Mathematikgenie selbst erklärte die von ihm gefundenen Formeln als Eingebungen der lokalen Göttin Namagiri. Nach Ramachandran sind Savants „die lebende Widerlegung der Behauptung, dass Spezialbegabungen nur eine gezielte Entfaltung der allgemeinen Intelligenz seien“. ${ }^{25}$ Fast jedes Cenie habe etwas von einem Savant an sich: hochbegabt in einigen wenigen Bereichen, aber völlig durchschnittlich in allen anderen. ${ }^{26}$

Norman Geschwind und Albert Galaburda (1987) haben festgestellt, dass „rechtshemisphärische Hochbegabung“, also das besondere räumliche Vorstellungsvermögen, das einer Hochbegabung in Mathematik, Musik und bildender Kunst zugrunde liegt, mit Nicht-Rechtshändigkeit (Links- oder Beidhändigkeit), Sprachproblemen (Dyslexie, Stottern, verspäteter Spracherwerb), Autismus und Autoimmunkrankheiten korreliert ist. Die gemeinsame Ursache dieser Eigenschaften nehmen Geschwind und Galaburda in einem erhöhten Testosteronspiegel in utero nach der zwanzigsten Schwangerschaftswoche an. ${ }^{27}$

Nach der Hypothese von Norman Geschwind und Albert Galaburda ${ }^{28}$ basieren die paradoxen Eigenschaften von Savants auf einer sog. Superioritätspathologie, d.h. dass beim Fötus eine mangelhafte Entwicklung bestimmter Hirnbereiche ein kompensatorisches Wachstum bestimmter anderer Bereiche auslöst. Bei Savants führe ein verlangsamtes Hirnwachstum in bestimmten Arealen der linken Hemisphäre zu einer Vergrößerung zum einen der kontralateralen kortikalen Areale (also in der rechten Hemisphäre), zum anderen benachbarter, nicht-betroffener Areale der linken Hemisphäre. Die Hypertrophie bestimmter Hirnareale ist demnach die Ursache der extremen Fähigkeiten von Savants; die mangelhafte Entwicklung anderer die Ursache ihrer Retardierung, ihrer Sprachprobleme sowie ihrer geringen allgemeinen Intelligenz. Die These des kompensatorischen Hirnwachstums als Folge von Entwicklungsverzögerungen oder Läsionen während der Embryonalphase ist im Tierversuch bestätigt worden. Geschwind und Galaburda stützen sich u.a. auf den 1918 von F. Sano beschriebenen Fall eines bildenden Künstlers mit gravierender, vor allem sprachlicher Retardierung: Bei der Obduktion zeigte sich, dass er sehr kleine Frontal- und Temporallappen, aber enorm entwickelte Occipitalareale hatte. ${ }^{29}$

Die Rechenbegabung bestimmter Savants lässt sich nach Geschwind und Galaburda folgendermaßen erklären: Entwicklungsverzögerungen in Teilen der linken Hemisphäre, möglicherweise durch eine hohe Testosteronkonzentration in utero, führen zu hypertrophem Wachstum der rechten Hemisphäre, aber auch bestimmter Bereiche der linken Hemisphäre, insbesondere sol-

25 Vgl. Ramachandran/Blakeslee (2002), S. 311. 26 Vgl. Ramachandran/Blakeslee (2002), S. 313.

27 Vgl. Winner (1998), S. 151-163.

$28 \mathrm{Vgl.} \mathrm{Geschwind/Galaburda} \mathrm{(1987),} \mathrm{S.} \mathrm{95-102.}$

$29 \mathrm{Vgl}$. Geschwind/Galaburda (1987), S. $101 \mathrm{f}$. 
cher, die für das Rechnen zuständig sind. Daraus könnten Sprachprobleme, aber auch außergewöhnliche visuell-räumliche Fähigkeiten sowie extreme Rechenfähigkeiten resultieren. Möglich sei auch, dass Savant-Rechenkünstler rechtshemisphärische, visuell-räumliche Strategien benutzen, also Zahlen und deren Strukturen sehen, statt damit zu rechnen.

Das Geschwind-Galaburda-Modell sagt für Savants erstens eine Neigung zur Nicht-Rechtshändigkeit, zweitens Störungen des Immunsystems, drittens messbare linksseitige Hirnanomalien voraus. Für die ersten beiden Hypothesen ist eine Bestätigung wahrscheinlich, denn Savants sind meist Autisten, und diese haben eine Neigung zu Nicht-Rechtshändigkeit sowie zu Autoimmunkrankheiten. Für die dritte Hypothese gibt es ebenfalls Belege: So haben CT-Aufnahmen deutliche Hinweise auf linksseitige Schädigungen von SavantGehirnen, insbesondere bei Savant-Musikern, ergeben. ${ }^{30}$

Für die These einer frühen Hirnschädigung von Savants spricht unter anderem die von Darold Treffert festgestellte Tatsache, dass sich bei SavantsMusikern häufig die Triade Blindheit, Retardierung und Musikalität findet als Folge von Frühgeburtlichkeit und künstlicher Beatmung mit zu hoher Sauerstoffkonzentration, was neben den kognitiven Schäden auch zu Blindheit durch rentrolaterale Fibroplasie führt. Die Musikalität beschränkt sich allerdings auf die Fähigkeit, gehörte Stücke exakt zu reproduzieren (meist auf dem Klavier), wobei dies mechanisch, stereotyp und ohne emotionale Beteiligung geschieht und keinerlei Innovationen hervorbringt. ${ }^{31}$ Bei einer prä- oder perinatalen Schädigung der linken Hemisphäre kann die rechte Hemisphäre noch kompensatorisch wachsen, was in späterem Alter nicht mehr möglich ist. Das phänomenale Gedächtnis, die emotionale Distanz und die äußerst konzentrierte, eng begrenzte Aufmerksamkeit von Savants könnten aus einer Verletzung des Cortexbereichs resultieren, der für ein bewusstes, bedeutungsvolles Erinnerungsvermögen erforderlich ist, so dass das Gedächtnis auf eine primitivere, unbewusste, automatische, vom kortiko-striatalen System vermittelte Stufe zurückfälltt. ${ }^{32}$

Auch Ramachandran nimmt an, dass bei Savants bestimmte spezialisierte Gehirnregionen auf Kosten anderer vergrößert worden sind und dass am Anfang eine prä- oder perinatale Hirnschädigung steht, der eine Umkartierung des Gehirns folgt (ähnlich wie beim Entstehen von Phantomgliedern nach Amputationen), so dass andere Bereiche größer und dichter an Neuronen werden. Ramachandran nimmt an, dass die extremen mathematischen Fähigkeiten einiger Savants auf einem hypertrophen Wachstum des Gyrus angularis in der linken Hirnhemisphäre basieren. Das Funktionieren dieser Region ist eine notwendige Voraussetzung für die Fähigkeit zum Rechnen, wie von Patienten mit Läsionen in diesen Bereichen bekannt ist. Eine Verdopplung der Größe des linken Gyrus angularis würde wahrscheinlich nicht bloß zu einer Verdopplung der mathematischen Fähigkeiten führen, sondern zu deren logarithmischer

30 Vgl. Winner (1998), S. 160-163.

31 Vgl. Treffert (1989), S. 34 und 204; Fallbeispiele auf den Seiten 103-122 und 187-193.

32 Vgl. Winner (1998), S. 160-163; Geschwind/Galaburda (1987); Treffert (1989). 
Zunahme. Dasselbe könnte für den rechten Gyrus angularis und zeichnerische Fähigkeiten gelten. ${ }^{33}$

Die Hypothese der Superioritätspathologie führt also das Savant-Syndrom auf eine prä- oder perinatale Schädigung bestimmter Hirnregionen und eine Überkompensation durch das hypertrophe Wachstum bestimmter anderer Hirnregionen zurück. Die frühen Hirnschädigungen können durch Testosteronüberschuss in einer bestimmten Schwangerschaftsphase, durch Sauerstoffmangel während der Geburt oder durch übermäßige Sauerstoffzufuhr bei Frühgeborenen entstehen. Diese Hypothese erklärt auch, warum es viel mehr männliche als weibliche Savants gibt: Zum einen sind männliche Föten viel höheren Testosteronkonzentrationen ausgesetzt, zum anderen entwickeln sich deren Gehirne langsamer und sind damit verletzungsanfälliger. Die Hypothese erklärt auch, warum sich Savants nur in bestimmten Domänen finden, nämlich in solchen, die entweder durch die rechte Hemisphäre vermittelt werden (Zeichen, Musik) oder durch linkshemisphärische Regionen, die an die hintere (bei Savants geschädigte) Sprachregion angrenzen (Rechnen). ${ }^{34}$

Nach einer Hypothese von Synder und Mitchell (1999) haben Savants Zugang zu den frühen Schritten der Informationsverarbeitung. Anna Milena Dubischar-Krivec (2006) hat diese These mit Hilfe einer magnetencephalographischen (MEG) Untersuchung mit kombinierter elektroencephalographischer Ableitung (EEG) geprüft. Demnach hat die Fähigkeit zum Kalenderrechnen bei autistischen Savant-Kalenderrechnern andere Grundlagen als dieselbe Fähigkeit bei geübten, nicht-autistischen Kalenderrechnern: Während bei ersteren bei der Berechnung von Daten aus Vergangenheit und Gegenwart vermehrt Gedächtnisprozesse beteiligt zu sein scheinen, basiert die Fähigkeit des Kalenderrechnens bei den Nichtautisten eher auf der expliziten Kenntnis von arithmetischen Gesetzmäßigkeiten und der Anwendung regelbasierter Strategien. ${ }^{35}$

Es gibt auch einige Fälle eines erworbenen Savant-Syndroms. In einem Fall wurde ein neunjähriger Junge nach einer Schussverletzung in seiner linken Schläfe taubstumm und schwerbehindert; gleichzeitig erlangte er jedoch eine enorme technische Begabung. In dem berühmt gewordenen Fall des Orlando Serrell wurde dieser als Zehnjähriger unmittelbar nach einer Kopfverletzung durch einen Baseball zu einem Kalenderrechner. Auch bei manchen älteren Menschen mit frontotemporaler Demenz tritt nach Beobachtungen von Bruce Miller das Savant-Syndrom auf: Zu Beginn der Krankheit entsteht plötzlich eine Inselbegabung: bei einigen das absolute Gehör, bei anderen eine hervorragende Zeichenbegabung. Robyn Young hat bei 17 gesunden Probanden ein passageres Savant-Syndrom hervorgerufen, indem er mittels repetitiver transkranieller magnetischer Stimulation (rTMS) die Areale im linken Schläfenlappen stimulierte, die bei Patienten mit erworbenem Savant-Syndrom geschädigt sind. Fünf Probanden zeigten während der Stimulation plötzlich Savant-artige Fähigkeiten: Kalenderrechnen bzw. Zeichenkunst. Ähnliche Untersuchungen haben Allan Synder und John Mitchell durchgeführt. Nach

33 Vgl. Ramachandran/Blakeslee (2002), S. 283-320.

34 Vgl. Treffert (1989), S. 195-198; Winner (1998), S. 163; Ramachandran/Blakeslee (2002), S. 283-320.

35 Vgl. Dubischar-Krivec (2006). 
ihrer These bewirkt die Stimulation, dass man die ,Rohdaten ' der Welt sieht, quasi ungefiltert, so wie sie im Unbewussten eines jeden Menschen repräsentiert sind. ${ }^{36}$ Bei Savants sei die oberste Ebene der mentalen Prozesse, also das konzeptionelle Denken und das Schlussfolgern, gestört, und dadurch arbeiteten die Prozesse der unteren Ebene mit großer Exaktheit und Detailtreue, da unbeeinflusst durch Abstraktion. Dies ist die Folge einer erworbenen Schädigung oder einer durch rTMS verursachten passageren Minderdurchblutung des Frontotemporallappens, bei der sowohl die Sprachfähigkeit als auch die Fähigkeit, Gesichter zu erkennen, verloren gehen. ${ }^{37}$

\section{Künstliche Erschaffung von Hochbegabten oder von Savants ${ }^{38}$}

In Zukunft wird es wahrscheinlich medizintechnische Möglichkeiten zur Erzeugung hochbegabter Kinder geben.

Die biotechnische Steigerung der Intelligenz fängt bei der gezielten Auswahl der biologischen Eltern an. Da Intelligenz ein erbliches Merkmal ist, besteht die einfachste Methode, ein intelligentes Kind zu produzieren, darin, eine Eizelle einer intelligenten Frau mit einer Samenzelle eines intelligenten Mannes zu verschmelzen. Dies geschieht immer schon auf natürliche Weise durch freiwillige Partnerwahl, kann aber mit modernen medizintechnischen Mitteln auch gezielt unternommen werden. Während zahlreiche Samenbanken die Möglichkeit bieten, genetisches Material nach den Kriterien IQ und Beruf des Spenders auszuwählen, ${ }^{39}$ sind käufliche Eizellen von intelligenten Frauen Mangelware, denn Eizellentnahmen erfordern die hormonelle Hyperstimulation der Eierstöcke (mit erhöhtem Eierstockkrebsrisiko) und Operationen unter Vollnarkose. Das internationale Wohlstandsgefälle hat aber schon einen halblegalen Markt für Eizellen und einen Eizelltourismus nach Osteuropa entstehen lassen, ${ }^{40}$ während in den USA vor allem Studentinnen zum Verkauf ihrer Eizellen angeworben werden. ${ }^{41}$

Viele Menschen haben den Wunsch, einerseits genetisch eigenen Nachwuchs zu bekommen, andererseits hochintelligente Kinder zu haben. Für dieses Dilemma könnte die gentechnische Manipulation ihrer Keimzellen oder ihres Embryos verlockend erscheinen. Da für die Intelligenz eine Vielzahl von Genen relevant ist, und da noch weitgehend unerforscht ist, welche Gene die kognitiven Fähigkeiten beeinflussen, sind gezielte gentechnische Manipulationen zur Intelligenzsteigerung derzeit noch weit von der Anwendung bei Menschen entfernt. Im Tierversuch ist damit aber schon begonnen worden..$^{42}$ Dabei haben sich allerdings unerwartete Zusammenhänge gezeigt, die zur größten Vorsicht bei genetischen Manipulationen mit dem Ziel der In-

36 Vgl. Hein (2003); Snyder et al. (2003); Osborne (2003). Letzerer gibt einen Erfahrungsbericht über eine transkranielle Magnetstimulation bei Synder, bei der er plötzlich in der Lage war, naturgetreu Katzen zu zeichnen.

$37 \mathrm{Vgl.} \mathrm{Fox} \mathrm{(2007).}$

38 Vgl. zu diesem Kapitel Müller (2007) und (2008).

39 Vgl. Plotz (2005).

40 Vgl. Kraske/Ludwig (2005).

41 Vgl. Plotz (2005), S. 202.

42 Vgl. Tang et al. (1999); Falk (2001); Hawasli (2007); Hilbert (2007). 
telligenzerhöhung mahnen sollten: So traten bei Mäusen, deren Intelligenz gentechnisch gesteigert wurde, gehäuft chronische Schmerzen bei Entzündungen und Verletzungen auf. ${ }^{43}$ Offenbar gehören das Gedächtnis für bewusste Erinnerungen und das Schmerzgedächtnis zusammen. Bei Mäusen, deren Cdk5-Gen abgeschaltet wurde, verbesserte sich ebenfalls die Lernfähigkeit; der Grund dafür ist wahrscheinlich, dass diese Mäuse gegenüber ihrer Umgebung viel empfindlicher reagieren als normale Mäuse. ${ }^{44}$ Schon diese beiden Studien deuten darauf hin, dass mit unerwünschten Nebenwirkungen zu rechnen ist, wenn man ein so komplexes genetisches Merkmal wie die Intelligenz manipuliert.

Eine weitere Möglichkeit der pränatalen Intelligenzsteigerung ist die Injektion von bestimmten Hormonen oder Morphogenen in das Gehirn eines Embryos, um dieses zu außerordentlichem Wachstum anzuregen..$^{45}$ Die Größe des Gehirns lässt sich allerdings nicht steigern, ohne nicht gleichzeitig das Schädelvolumen zu vergrößern. Kinder mit extragroßen Gehirnen und extragroßen Schädeln müssten per Kaiserschnitt entbunden werden.

Statt die Größe des gesamten Gehirns zu manipulieren, könnte man versuchen, selektiv einzelne Gehirnbereiche zu hypertrophem Wachstum anzuregen, um spezielle Hochbegabungen zu erzeugen: Ein mathematisch hochbegabtes Kind könnte man vielleicht dadurch erzeugen, dass man nach der zwanzigsten Schwangerschaftswoche den Testosteronspiegel in utero erhöht, um dadurch das Wachstum der linken Hemisphäre zu behindern und das Wachstum der rechten Hemisphäre zu begünstigen. Eine solche Maßnahme ließe auf ein Kind mit einem besonders guten räumlichen Vorstellungsvermögen und einer Hochbegabung in Mathematik, Musik und bildender Kunst hoffen. Allerdings müsste man auch mit Sprachproblemen (Dyslexie, Stottern, verspäteter Spracherwerb), Autismus und Autoimmunkrankheiten rechnen. ${ }^{46}$ Um ein zeichnerisch hochbegabtes Kind zu erschaffen, müsste man ein Kind mit einem hypertrophen rechten Gyrus angularis erzeugen.$^{47}$ Zur Zeit ist noch nicht viel darüber bekannt, welche hirnorganischen Besonderheiten einer besonders hohen allgemeinen Intelligenz bzw. den verschiedenen Spezialbegabungen zu Grunde liegen, und daher gibt es auch noch keine etablierten Methoden, um Hochbegabungen im Embryonalstadium zu erschaffen. Jede Steigerung wird auf der einseitigen Vergrößerung bestimmter Gehirnbereiche und damit auf der Verkleinerung anderer basieren, so dass die Steigerung bestimmter kognitiver Funktionen wahrscheinlich die Verschlechterung anderer Funktionen mit sich bringen wird. Dabei werden wahrscheinlich eher Idiots

43 Tang, Wei, Zhuo et al. von der Washington University School of Medicine haben Mäuse gentechnisch so verändert, dass eine Überexpression eines NMDA-Rezeptors in den Vorderhirnen der Mäuse stattfand, die zur verbesserten Aktivierung der NMDA-Rezeptoren führte, wodurch die synaptischen Potentiale als Antworten auf Reize erleichtert wurden. Diese Mäuse zeigen verbesserte Lern- und Gedächtnisfähigkeiten. Sie entwickeln aber nach Entzündungen und Verletzungen auch stärkere chronische Schmerzen als unveränderte Artgenossen, wohingegen ihre Reaktion auf akute Schmerzen normal war. - Vgl. Tang et al. (1999); Wei et al. (2001); Falk (2001); Hilbert (2007).

44 Vgl. Hawasli (2007); Hilbert (2007).

$45 \mathrm{Vgl.} \mathrm{Ramachandran/Blakeslee} \mathrm{(2002),} \mathrm{S.} 318$.

46 Vgl. zur linkshemisphärischen Hochbegabung Winner (1998), S. 151-160.

$47 \mathrm{Vgl}$. Ramachandran/Blakeslee (2002), S. 317. 
savants als Genies erzeugt. Auf massive Behinderungen körperlicher, psychischer oder intellektueller Art sollte man bei derartigen Manipulationen gefasst sein.

Derartige Eingriffe in die Entwicklung von Embryonen oder Föten sind aus ethischer Sicht unbedingt abzulehnen, da sie gravierende, höchst riskante Manipulation an einer werdenden Person sind, deren Einverständnis dazu kaum vorausgesetzt werden kann. Gezielte Experimente dieser Art sind noch Spekulation. Trotzdem ist damit zu rechnen, dass solche Experimente jetzt oder in naher Zukunft irgendwo auf der Welt durchgeführt werden.

\section{Literatur}

Brackmann (2005) Andrea Brackmann, Jenseits der Norm - hochbegabt und hoch sensibel?, Stuttgart 2005

Csikszentmihalyi (1996): Mihaly Csikszentmihalyi, Kreativität. Wie Sie das Unmögliche schaffen und Ihre Grenzen überwinden, Stuttgart 1996

Deary/Der (2005): Ian I. Deary, Geoff Der, Reaction Time Explains IQ's Association With Death, Psychological Science 16 (2005), 1, p. 64-69

Dubischar-Krivec (2006): Anna Milena Dubischar-Krivec, Neurophysiologie des Kalenderrechnens bei autistischen Savants und geübten Experten, Dissertation, Tübingen 2006

Eysenck (1995): Hans Jürgen Eysenck, Genius. The natural history of creativity, Cambridge 1995

Falk (2001): Marcel Falk, Hypergescheite Gentech-Mäuse von chronischen Schmerzen geplagt, Bild der Wissenschaft Online, 02.02.2001

Feger (1988): Barbara Feger, Hochbegabung. Chancen und Probleme, Bern 1988

Feger/Prado (1998): Barbara Feger, Tanja Prado, Hochbegabung. Die normalste Sache der Welt, Darmstadt 1998

Fortenbacher (2006): Astrid Fortenbacher, Hochbegabung bei Vor- und Grundschulkindern. Verhaltensmerkmale, Risiken, Förderung, Saarbrücken 2006

Fox (2002): Douglas S. Fox, The Inner Savant, Brain \& Mind, 02.01.2002, http://discovermagazine.com/2002/ feb/featsavant/ [09.07.2007]

Freund-Braier (2000): Inez Freund-Braier, Hochbegabung, Hochleistung, Persönlichkeit, Münster 2000

Gardner (1993): Howard Gardner, Creative mind, New York 1993

Geschwind/Galaburda (1987): Norman Geschwind, Albert M. Galaburda, Cerebral lateralization, Cambridge, London 1987

Goleman (1998): Daniel Goleman, Emotionale Intelligenz, München, 8. Aufl., 1998

Gould/Grant Gould: James L. Gould, Carol Grant Gould, Bewußtsein bei Tieren. Ursprünge von Denken, Lernen und Sprechen, Heidelberg et al. 1997

Guthke (1996): Jürgen Guthke, Intelligenz im Test. Wege der psychologischen Intelligenzdiagnostik, Göttingen, Zürich 1996

Hagner (2007): Michael Hagner, Geniale Gehirne. Zur Geschichte der Elitegehirnforschung, München 2007

Hawasli et al. (2007): Ammar Hawasli et al., Cyclin-dependent kinase 5 governs learning and synaptic plasticity via control of NMDAR degradation, Nature Neuroscience, 27.05.2007

Hein (2003): Till Hein, Das geheime Wissen der Erbsenzähler, Die Zeit 30 (2003), http://images.zeit.de/text/ 2003/30/M-Autismus [09.07.2007]

Hilbert (2007): Claudia Hilbert, Wie man Mäuse schlauer macht. Ausschalten des Cdk5-Gens verbessert die Lernfähigkeit der Tiere, Bild der Wissenschaft Online, 30.05.2007

Howe (2001): Michael Howe, Genius Explained, Cambridge 2001

Klimesch/Schimke (1998): Wolfgang Klimesch, Hannes Schimke, Psychophysiologische Voraussetzungen von Intelligenzleistungen, in: Erwin Roth (Hrsg.), Intelligenz. Grundlagen und neuere Forschung, Stuttgart 1998, S. 144-160 
Kraske/Ludwig (2005): Marion Kraske, Udo Ludwig, Die Babygrenze. Wie deutsche Frauen zur Befruchtung ins Ausland reisen, Der Spiegel 46 (2005), www.spiegel.de/spiegel/0,1518,384583,00.html, 09.04.06

Kretschmer (1929): Ernst Kretschmer, Geniale Menschen, Berlin 1929

Lange-Eichbaum (2000): Wilhelm Lange-Eichbaum, Genie, Irrsinn und Ruhm. Die geheimen Psychosen der Mächtigen, Köln 2000

Miller (2001): Geoffrey Miller, Die sexuelle Evolution. Partnerwahl und die Entstehung des Geistes, Heidelberg, Berlin 2001

Müller (2004): Sabine Müller, Programm für eine neue Wissenschaftstheorie, Würzburg 2004

Müller (2006): Sabine Müller, Intelligenz-Theorien der Philosophie und der empirischen Wissenschaften, in: Dominik Groß, Tobias H. Duncker (Hrsg.) Farbe - Erkenntnis - Wissenschaft. Zur epistemischen Relevanz von Farbe in der Medizin, Münster 2006, S. 145-154

Müller (2007): Sabine Müller, Ist Cognitive Enhancement zur Steigerung der Intelligenz ethisch geboten? Diskussion utilitaristischer und idealistischer Argumente, in: Joachim Ach, Uwe Opolka, Bettina SchöneSeifert et al. (Hrsg.), Neuro-Enhancement. Ethik vor neuen Herausforderungen, Paderborn 2007

Müller (2008): Sabine Müller, Intelligenztheorien der Medizin und der Naturwissenschaften - eine wissenschaftshistorische und ethische Studie, in Vorbereitung, 2008

Müller/Steinmetzer (2007): Sabine Müller, Jan Steinmetzer, Kulturgeschichte der Hochbegabung - Zwischen Pathologisierung und Idealisierung, in: Dominik Groß, Florian Steger (Hrsg.), Proceedings-Band zu den Kick-Off-Workshops am 12.10., 26.10. und 20.11.2006, Aachen 2007, S. 101-103

Nissen (2005): Gerhardt Nissen, Kulturgeschichte seelischer Störungen bei Kindern und Jugendlichen, Stuttgart 2005

Oehme (1988): Johannes Oehme, Das Kind im 18. Jahrhundert. Beiträge zur Sozialgeschichte des Kindes, Lübeck 1988

Osborne (2003): Lawrence Osborne, Savant for a Day, New York Times Magazine, 22.06.2003, www.wireheading. com/brainstim/savant.html [23.03.2006]

Plotz (2005): David Plotz, The Genius Factory. Unravelling the mysteries of the Nobel Price Sperm Bank, London 2005

Pruisken (2005): Christiane Pruisken, Interessen und Hobbys hochbegabter Grundschulkinder, Münster 2005

Ramachandran/Blakeslee (2002): Vilayanur Ramachandran, Sandra Blakeslee, Die blinde Frau, die sehen kann. Rätselhafte Phänomene unseres Bewusstseins, Reinbek 2002

Reichle/Lehmann/Jüling (2004): Barbara Reichle, Wolfgang Lehmann, Inge Jüling, Hochbegabte Kinder. Erkennen, fördern, problematische Entwicklungen verhindern, Weinheim 2004

Ross (2007): Philip E. Ross, Wie Genies denken, Spektrum der Wissenschaft 1 (2007), S. 36-43

Rost (1993): Detlef H. Rost (Hrsg.), Lebensumweltanalyse hochbegabter Kinder. Das Marburger Hochbegabtenprojekt, Göttingen 1993

Rudinger/Rietz (1997): Georg Rudinger, Christian Rietz, Survival of the brightest? Über den Zusammenhang zwischen Intelligenz und Lebenserwartung, Forschung und Lehre 2 (1997), S. $68 \mathrm{f}$.

Sacks (1990): Oliver Sacks, Der Mann, der seine Frau mit einem Hut verwechselte, Reinbek 1990

Schilling (2002): Susanne R. Schilling, Hochbegabte Jugendliche und ihre Peers. Wer allzu klug ist, findet keine Freunde?, Münster 2002

Schulte zu Berge (2001): Sabine Schulte zu Berge, Hochbegabte Kinder in der Grundschule. Erkennen - Verstehen - Im Unterricht berücksichtigen, Münster, 2. Aufl., 2001

Schütz (2004): Corinna Schütz, Leistungsbezogenes Denken hochbegabter Kinder, Münster 2004

Snyder/Mitchell (1999): Allan W. Snyder, D. John Mitchell, Is integer arithmetic fundamental to mental processing?: the mind's secret arithmetic, Proceedings of the Royal Society of London, Series B, 266 (1999), p. 587-592

Snyder et al. (2003): Allan W. Snyder, E. Mulcahy, I. L. Taylor et al., Savant-like skills exposed in normal people by suppressing the left fronto-temporal lobe, Journal of Integrative Neuroscience 2 (2003), 2, p. 149-158

Sparfeldt (2006): Jörn R. Sparfeldt, Berufsinteressen hochbegabter Jugendlicher, Münster 2006 
Storch (1751), Johann Storch, Theoretische und Practische Abhandlung von Kinderkranckheiten, Band 4, Eisenach 1751

Tang et al. (1999): Ya-Ping Tang, Eiji Shimizu, Gilles R. Dube et al., Genetic enhancement of learning and memory in mice, Nature 401 (1999), 2.09.1999, p. 63-69

Treffert (1989): Darold A. Treffert, Extraordinary people, New York 1989

Tücke (2005) Manfred Tücke, Schulische Intelligenz und Hochbegabung, Münster 2005

Urban (2004): Klaus Urban, Hochbegabungen. Aufgaben und Chancen für Erziehung, Schule und Gesellschaft, Münster 2004

Vom Scheidt (2004): Jürgen vom Scheidt, Das Drama der Hochbegabten. Zwischen Genie und Leistungsverweigerung, München 2004

Waldmann/Weinert (1990): Michael Waldmann, Franz E. Weinert, Intelligenz und Denken. Perspektiven der Hochbegabungsforschung, Göttingen et al. 1990

Wei et al. (2001): Feng Wei, Guo-Du Wang, Geoffrey A. Kerchner et al., Genetic enhancement of inflammatory pain by forebrain NR2B overexpression, Nature Neuroscience 4 (2001) p. 164-169

Winner (1998): Ellen Winner, Hochbegabt. Mythen und Realitäten von außergewöhnlichen Kindern, Stuttgart 1998

Zimbardo (1995): Philip G. Zimbardo, Psychologie, Heidelberg, 6. Aufl., 1995 